\section{Audiology \\ Neurotology}

Audiol Neurotol 2017;22:125-134

DOI: $10.1159 / 000479218$
Received: September 30, 2016

Accepted: July 5, 2017

Published online: September 9, 2017

\title{
Brimonidine Protects Auditory Hair Cells from in vitro-Induced Toxicity of Gentamicin
}

\author{
Maurizio Cortada ${ }^{\mathrm{a}}$ Soledad Levano ${ }^{\mathrm{a}}$ Daniel Bodmer ${ }^{\mathrm{b}}$ \\ a Department of Biomedicine, University of Basel, and ${ }^{b}$ Clinic for Otolaryngology, Head and Neck Surgery, \\ University of Basel Hospital, Basel, Switzerland
}

\section{Keywords}

Hair cells · Organ of Corti - Brimonidine - Gentamicin · Toxicity

\begin{abstract}
Brimonidine, an alpha-2 adrenergic receptor $\left(a_{2}-A R\right)$ agonist, has neuroprotective effects in the visual system and in spiral ganglion neurons. Auditory hair cells (HCs) express all $3 \mathrm{a}_{2^{-}}$ AR subtypes, but their roles in HCs remain unknown. This study investigated the effects of brimonidine on auditory $\mathrm{HCs}$ that were also exposed to gentamicin, which is toxic to $\mathrm{HCs}$. Organ of Corti explants were exposed to gentamicin in the presence or absence of brimonidine, and the $a_{2}$-AR protein expression levels and Erk1/2 and Akt phosphorylation levels were determined. Brimonidine had a protective effect on auditory $\mathrm{HCs}$ against gentamicin-induced toxicity that was blocked by yohimbine. This suggested that the protective effect of brimonidine on HCs was mediated by the $a_{2}-A R$. None of the treatments altered $\mathrm{a}_{2}$-AR protein expression levels, and brimonidine did not significantly change the activation levels of the Erk1/2 and Akt proteins. These observations indicated that brimonidine, acting directly via $a_{2}-A R$, protects HCs from gentamicin-induced toxicity. Therefore, brimonidine shows potential for preventing or treating sensorineural hearing loss.

(C) 2017 The Author(s)

Published by S. Karger AG, Basel
\end{abstract}

\section{KARGER}

E-Mail karger@karger.com www.karger.com/aud
2017 The Author(s)

Published by S. Karger AG, Basel

Karger

0 pen access

This article is licensed under the Creative Commons AttributionNonCommercial-NoDerivatives 4.0 International License (CC BYNC-ND) (http://www.karger.com/Services/OpenAccessLicense). Usage and distribution for commercial purposes as well as any distribution of modified material requires written permission.

\section{Introduction}

According to the WHO, there are 360 million people in the world with disabling hearing loss [World Health Organization, 2012]. While conductive hearing loss can be treated, sensorineural hearing loss due to the loss of auditory hair cells (HCs) cannot. Furthermore, the loss of auditory HCs is irreversible in mammals. Therefore, it is important to better understand the molecular mechanisms and pathways involved in HC death and survival in order to develop preventive and therapeutic strategies.

Brimonidine, also known as UK-14304, is a highly selective alpha-2 adrenergic receptor $\left(\alpha_{2}-\mathrm{AR}\right)$ agonist that has shown neuroprotective effects in the visual system [Kim et al., 2007; Knels et al., 2008; Lai et al., 2002] and on spiral ganglions [Cai et al., 2013a]. There are $3 \alpha_{2}$-AR subtypes, namely $\alpha_{2 a}, \alpha_{2 b}$, and $\alpha_{2 c}$, that mediate the physiological activity of adrenaline and noradrenaline. All 3 receptor subtypes are part of the $G_{i} / G_{0}$ subfamily of $\mathrm{G}$ protein-coupled receptors. These receptors inhibit the activity of adenylyl cyclase, which in turn inhibits the opening of voltage-gated calcium channels, thereby opening potassium channels and stimulating phospholipase $\mathrm{C}$, phosphatidylinositide 3-kinase (PI3 kinase), and the mitogen-activated protein kinase (MAPK) pathway [Daunt et al., 1997; Hawes et al., 1995; MacDonald et al., 1997].

Daniel Bodmer

Clinic for Otolaryngology, Head and Neck Surgery University of Basel Hospital

Petersgraben 4, CH-4031 Basel (Switzerland)

E-Mail daniel.bodmer@usb.ch 
All $3 \alpha_{2}$-AR subtypes are present in the neonatal rat cochlea and on inner HCs (IHCs) and outer HCs (OHCs) on postnatal (P) days P3 and P8 [Cai et al., 2013b]. In addition, the rat organ of Corti (OC) shows adrenergic innervation [Drescher et al., 2006]. Interestingly, experiments suggest that sympathetic fibers with noradrenaline as the postganglionic neurotransmitter affect the susceptibility to noise-induced hearing loss [Bielefeld and Henderson, 2007].

Although the mechanisms underlying the neuroprotective effects of brimonidine are not entirely understood, PI3 kinase-dependent pathways appear to be involved [Knels et al., 2008; Yukita et al., 2016]. Specifically, the PI3 kinase signaling pathway is involved in the HC survival of gentamicin-induced toxicity [Chung et al., 2006], and our group recently showed that protein kinase $\mathrm{B}$ (Akt) is involved in HC survival [Brand et al., 2015]. Notably, exposure to brimonidine can lead to Akt phosphorylation [Kim et al., 2007; Lai et al., 2002; Tatton et al., 2001], and brimonidine exerts its neuroprotective effects by activating the MAPK pathway [Fujita et al., 2013; Harun-OrRashid et al., 2014; Lai et al., 2002]. After exposure to brimonidine, there is phosphorylation of the extracellular signal-activated kinases 1/2 (Erk1/2) in injured retinal homogenates [Fujita et al., 2013] or as a physiological response to $\alpha_{2}$-AR activation in uninjured retinal homogenates [Lai et al., 2002] and chicken Müller cells [HarunOr-Rashid et al., 2014]. Phosphorylation of Erk1/2 has especially been shown to mediate HC survival [Bas et al., 2012; Kurioka et al., 2015], and one way to protect OHCs from gentamicin-induced toxicity is increased activation of MAPK/Erk [Battaglia et al., 2003].

In addition to these molecular mechanisms, brimonidine has also been shown to have neuroprotective properties via $\mathrm{N}$-methyl-D-aspartate (NMDA) inhibition [Dong et al., 2008; Han and Wu, 2002], growth factor upregulation [Gao et al., 2002; Kim et al., 2007; Lonngren et al., 2006], and involvement in apoptotic pathways [Cai et al., 2013a; Lai et al., 2002]. Furthermore, brimonidine inhibits protein kinase A [Grueb et al., 2008]. Interestingly, Navaratnam et al. [1996] demonstrated that the proliferation and regeneration of the avian auditory epithelium depends on the activation of protein kinase A, which is cAMP-dependent, and that inhibition of protein kinase A correlates with decreased regeneration.

Little is known about the role of the $\alpha_{2}$-AR in HCs. The neuroprotective effects of brimonidine on different cell types and the presence of all $3 \alpha_{2}$-AR subtypes in HCs prompted us to examine whether brimonidine could protect mammalian HCs from gentamicin-induced toxicity.
If we found a protective effect, we also wished to investigate whether it could be blocked by the $\alpha_{2}$-AR antagonist yohimbine. We further examined whether brimonidine changed the expression levels of the $\alpha_{2}$-AR protein. Finally, we investigated whether this drug could change Erk1/2 and Akt phosphorylation levels in OCs.

\section{Materials and Methods}

\section{Drugs and Antibodies}

Brimonidine tartrate (Y0001658), yohimbine hydrochloride (Y3125), gentamicin, and penicillin (SP600125) were obtained from Sigma-Aldrich Chemie GmbH (Steinheim, Germany). Primary antibodies against the following proteins were used at the indicated dilutions: phospho-p44/42 MAPK (p-Erk1/2)-Thr202/ Tyr204 (4370) and phospho-Akt-Ser473 (4060) at 1:1,000, and p44/42 MAPK (Erk1/2; 4695) and Akt (9272) at 1:500 (all from Cell Signaling, Bioconcept, Allschwil, Switzerland); GAPDH at 1:5,000 (ab8245; Abcam, Labforce AG, Nunningen, Switzerland), and $\alpha_{2 a}-A R$ at 1:500 (PA1-048; Affinity Bioreagents, Golden, CO, USA).

\section{OC Dissection}

All animal procedures were carried out in Basel, Switzerland, according to an animal research protocol that was approved by the Animal Care Committee of the Canton of Basel, Switzerland. All experiments were carried out with OCs from 5-day-old Wistar rat pups (Janvier Labs, Le Genest-Saint-Isle, France). The OCs were dissected from the skull and then transferred to cell culture plates with Dulbecco's modified Eagle medium supplemented with $10 \%$ fetal bovine serum, $25 \mathrm{mM}$ of HEPES, and $30 \mathrm{U} / \mathrm{mL}$ of penicillin, and incubated at $37^{\circ} \mathrm{C}$ in $5 \% \mathrm{CO}_{2}$.

\section{Tissue Culture}

$\mathrm{HC}$ damage was induced by exposure to $400 \mu \mathrm{M}$ gentamicin for $48 \mathrm{~h}$ for HC damage experiments, or for 8 or $24 \mathrm{~h}$ for the Western blot analyses. Before exposure to gentamicin, the OCs were pretreated with brimonidine $(1,10$, or $100 \mu \mathrm{M})$ and yohimbine $(10 \mu \mathrm{M})$ for 20-24 h. Yohimbine is an $\alpha_{2}-\mathrm{AR}$ antagonist that has been used to block the effects of brimonidine that are mediated by the $\alpha_{2}$-AR [Cai et al., 2013a; Harun-Or-Rashid et al., 2014, 2015; Lai et al., 2002]. The concentrations we used were based on a study that found that $10 \mu \mathrm{M}$ of yohimbine blocked the effects of several concentrations of brimonidine $(0.01-1 \mu \mathrm{M})$ [Cai et al., 2013a].

\section{HC Count}

After treatment, the OCs were fixed, permeabilized, and stained with Alexa Fluor 568 phalloidin (Invitrogen AG, Basel, Switzerland). The images were acquired using the Nikon A1R laser confocal microscope (Nikon AG Instruments, Egg, Switzerland). The surviving HCs were counted in a section corresponding to $20 \mathrm{IHCs}$ at different sites of the basal and middle turn of each OC in randomly selected fields. The apical turn was not used in our experiments since we observed almost no damage in the apex of the cochlea at our chosen gentamicin concentration. Previous experiments showed that the base of the cochlea is more susceptible to gentamicin damage than the apex [Sha et al., 2001]. The IHCs and 
Fig. 1. Brimonidine and yohimbine are not toxic to hair cells (HCs) in vitro. a Quantification of inner hair cells (IHCs) and outer hair cells (OHCs). b Representative images of phalloidin-stained HCs in organ of Corti (OC) explants exposed to the indicated concentrations of brimonidine. $n=5-6$ OCs per condition. c Quantification of IHCs and OHCs. d Representative images of phalloidin-stained HCs in OC explants exposed to yohimbine. $n=8$ OCs per condition. Scale bar for all figures, $20 \mu \mathrm{m}$. Data are expressed as the number of surviving HCs per 20 IHCs, counted at different sites for the basal and middle turn of each OC. Values are shown as means + SDs.

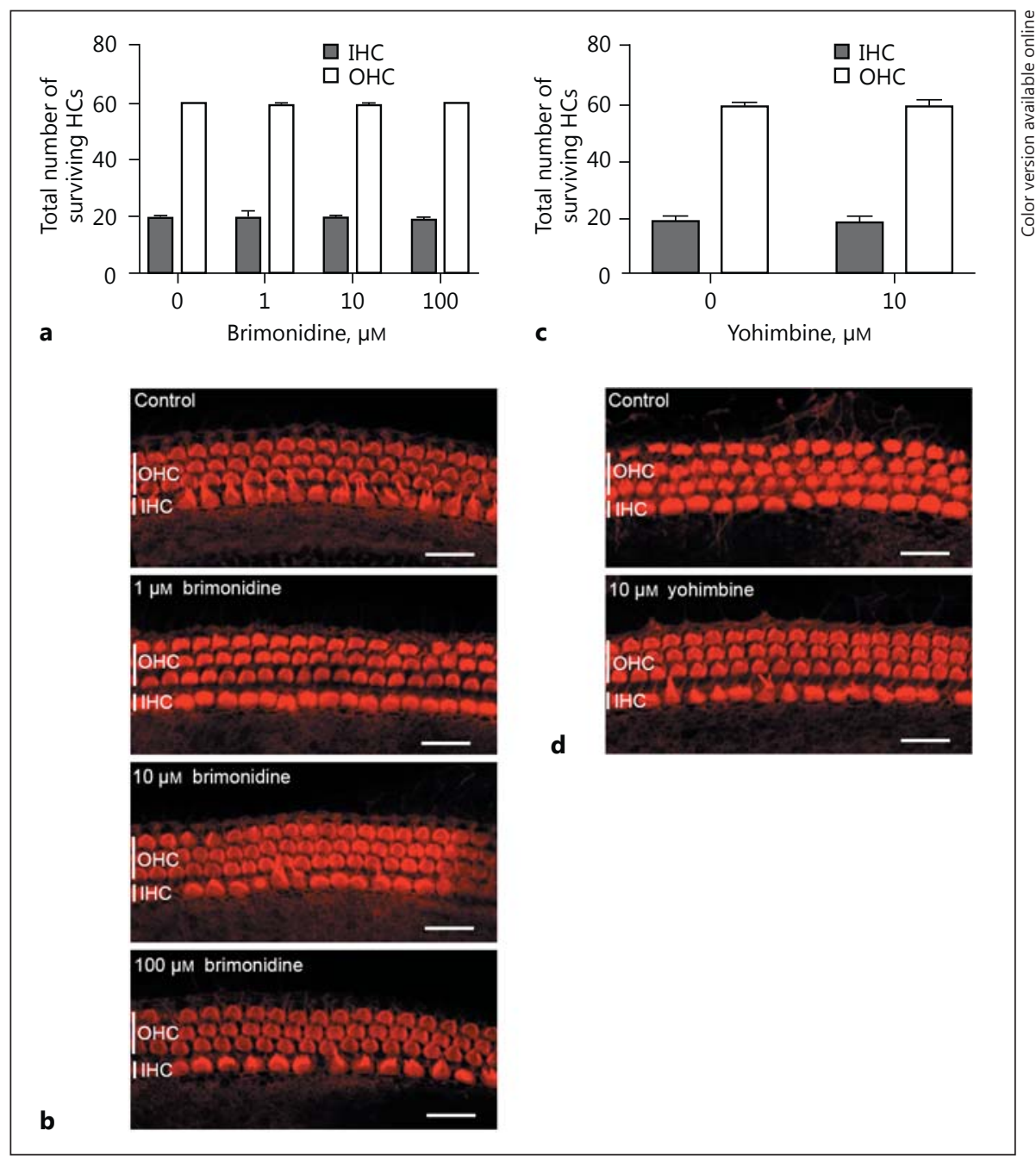

OHCs were counted to determine $\mathrm{HC}$ survival. If there was a gap in the normal ordered array of HCs, cells were considered to be missing because they had undergone apoptosis.

\section{Immunoblotting}

To prepare tissue lysate, the basal and middle turns of 6-7 OCs per experimental condition were dissected and cultured as described above. After treatment, the explant tissue was lysed with $20 \mu \mathrm{L}$ per OC of T-Per Tissue Protein Extraction Reagent (Thermo Fisher Scientific, LuBio Science, Lucerne, Switzerland) containing phosphatase/protease inhibitors (Roche, Indianapolis, IN, USA). Proteins were separated by electrophoresis and blotted onto a polyvinylidene difluoride membrane. The membrane blocking and antibody incubation steps were performed using the iBind Flex Western System (Thermo Fisher Scientific) according to the manufacturer's protocol. The bands were visualized via chemiluminescence using West Femto SuperSignal (Thermo Fisher Scientific). The blotted membranes were first used to visualize the phosphorylated forms of the proteins, then stripped with Restore PLUS Western Blot Stripping Buffer (Thermo Fisher Scientific) and reused to analyze the corresponding amounts of total protein. For each membrane, GAPDH was used as a loading control. Image J software (NIH) was utilized to calculate the relative densities of the specific protein bands for each condition. The amount of phospho-Erk1/2 and phospho-Akt relative to the total Erk and Akt protein was calculated. The ratio of the relative amount of $\alpha_{2 \mathrm{~A}}$-AR to GAPDH was also calculated. For the Western blot analysis, 3 biological replicates were used for the 24-h incubation, and 2 biological replicates were used for the 8-h incubation. An incubation time of $24 \mathrm{~h}$ was chosen because Erk1/2 activation was observed in previous studies after $24 \mathrm{~h}$ [Cai et al., 2013a; Harun-Or-Rashid et al., 2015]. Chung et al. [2006] observed Akt activation after exposure to gentamicin for $6 \mathrm{~h}$. We decided to investigate Akt activation after exposure to gentamicin for $8 \mathrm{~h}$.

\section{Statistical Analysis}

All data are presented as the mean \pm standard deviation. The statistical analysis was performed using Prism 6 (GraphPad software, La Jolla, CA, USA). We used 2-way ANOVA and the Tukey multiple comparisons test to analyze $\mathrm{HC}$ damage. For the protein ratios, we used the Kruskal-Wallis 1-way ANOVA test and Dunn multiple comparisons test. The results were considered statistically significant at $p<0.05$. 
Fig. 2. Brimonidine protects outer hair cells (OHCs) from gentamicin-induced toxicity. a Quantification of inner hair cells (IHCs) and OHCs in organ of Corti (OC) explants exposed to the indicated concentrations of gentamicin and brimonidine. $n=8$ OCs per condition. $\mathbf{b}$ Representative images of phalloidin-stained HCs. Exposure to increasing concentrations of brimonidine in addition to gentamicin decreased OHC loss compared with exposure to gentamicin alone. Scale bar for all figures, $20 \mu \mathrm{m}$. Data are expressed as the number of surviving HCs per 20 IHCs, counted at different sites for the basal and middle turn of each OC. Values are shown as means + SDs. ${ }^{* * * *} p<0.0001$ and ${ }^{*} p<$ 0.05 versus untreated control group.

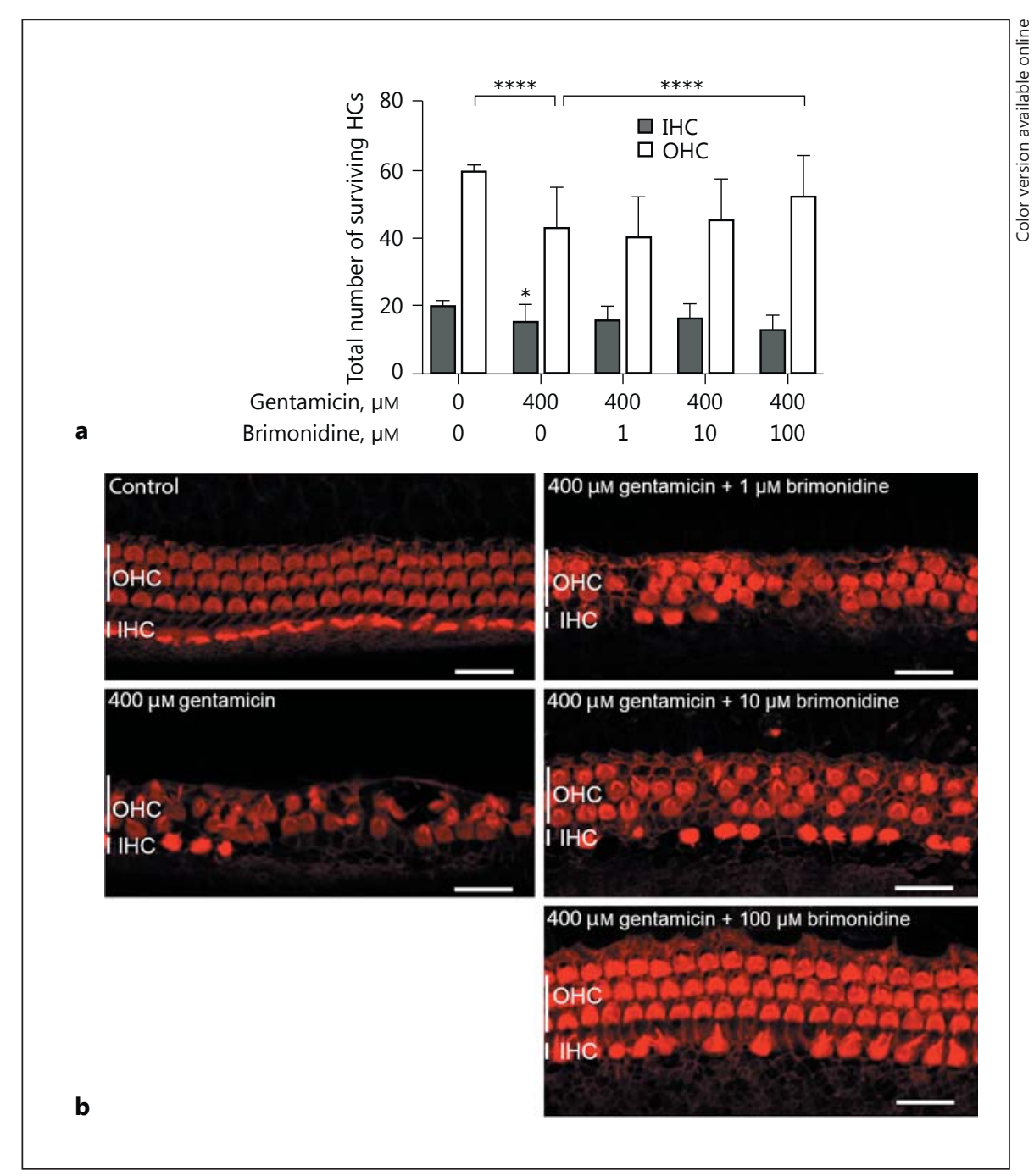

\section{Results}

\section{Brimonidine and Yohimbine Are Not Toxic to HCs in} vitro

To exclude possible toxic effects of brimonidine and yohimbine on HCs, OCs were incubated with the concentrations of brimonidine $(1,10,100 \mu \mathrm{M})$ and yohimbine $(10 \mu \mathrm{M})$ used in later experiments. There was no significant difference in cell number between the control group and OCs exposed to different concentrations of brimonidine (Fig. 1a). OC explants exposed to brimonidine alone showed 3 complete rows of OHCs and a complete row of IHCs, as in OCs treated with culture media alone (Fig. 1b). There was also no significant difference in the number of surviving HCs between the control group and OCs exposed to yohimbine (Fig. 1c). OC explants exposed to yo- himbine alone showed 3 orderly rows of OHCs and a single row of IHCs, as in the control group (Fig. 1d). Thus, brimonidine and yohimbine were not toxic to HCs.

\section{Brimonidine Protects OHCs from Gentamicin- \\ Induced HC Damage in vitro}

As expected, there was a significant loss of $\mathrm{OHCs}$ and IHCs after exposure to gentamicin (Fig. 2a). After $48 \mathrm{~h}$ of exposure to $400 \mu \mathrm{M}$ gentamicin, there were fewer surviving OHCs $(42.5 \pm 12.3)$ and IHCs $(14.6 \pm 5.4)$ compared to untreated OHCs $(59.4 \pm 2.1)$ and IHCs $(19.0 \pm 2.2)$ in a section of 80 HCs (Fig. 2a, b). The survival of OHCs in OCs exposed to gentamicin and $100 \mu \mathrm{M}$ brimonidine (51.8 $\pm 12.6)$ was significantly higher than in OCs exposed to gentamicin alone ( $42.5 \pm 12.3, p<0.0001$; Fig. 2a, b). 
Fig. 3. Yohimbine inhibits the protective effect of brimonidine on gentamicin-induced outer hair cell (OHC) damage in vitro. a Quantification of inner hair cells (IHCs) and OHCs in organ of Corti (OC) explants exposed to the indicated concentrations of gentamicin, brimonidine, and/ or yohimbine. $n=7-9$ OCs per condition. b Representative images of phalloidinstained hair cells (HCs). Compared to OCs exposed to gentamicin and brimonidine, those exposed to yohimbine in addition to gentamicin and brimonidine showed increased OHC loss, with more gaps in the normal ordered array of HCs. Scale bar for all figures, $20 \mu \mathrm{m}$. Data are expressed as the number of surviving HCs per 20 inner HCs, counted at different sites for the basal and middle turn of each OC. Values are shown as means + SDs. ${ }^{* * * *} p<0.0001$ and ${ }^{\#} p<0.0001$ versus untreated control groups.

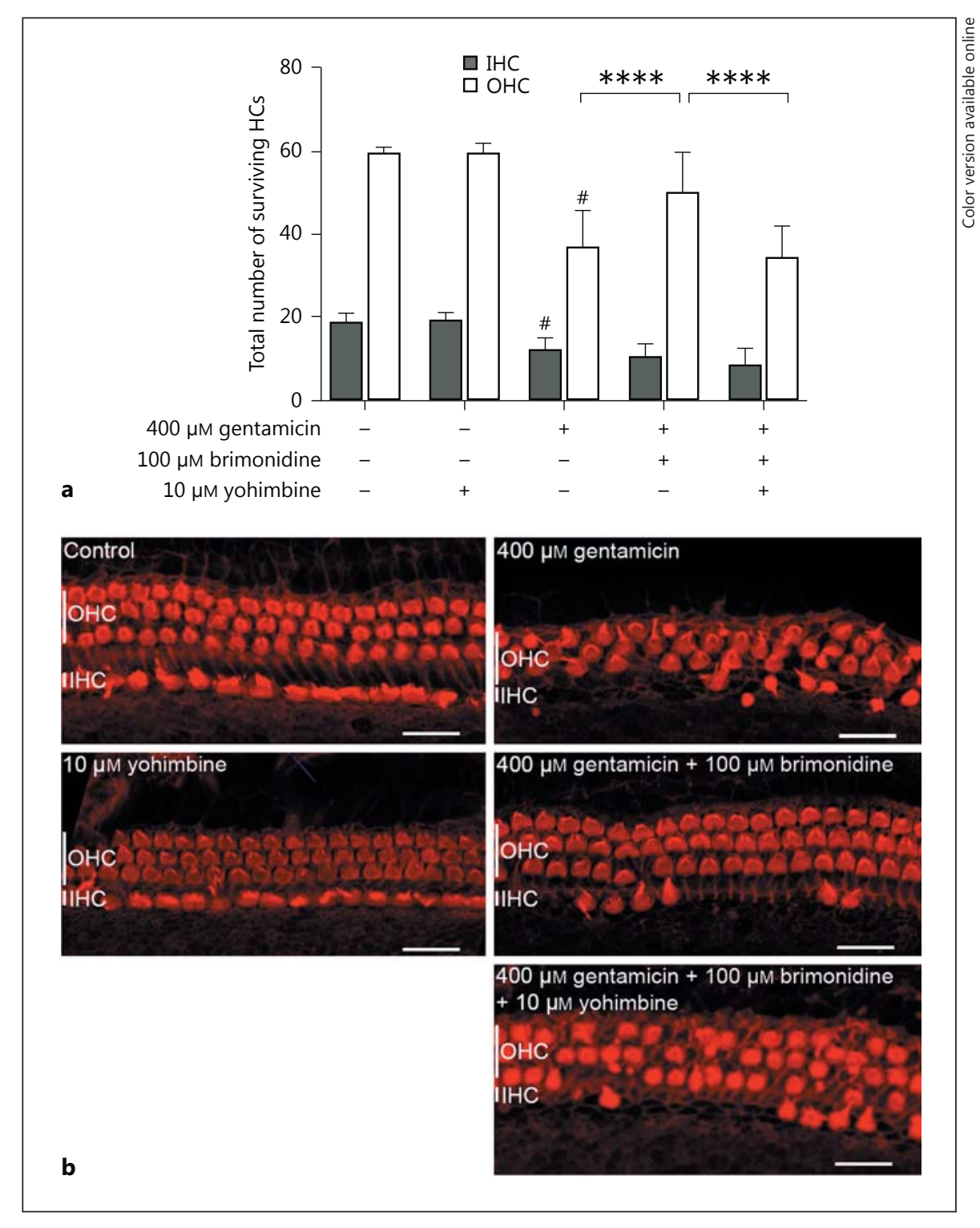

Yohimbine Inhibits the Protective Effect of

Brimonidine on Gentamicin-Induced OHC Damage in vitro

After exposure to gentamicin for $48 \mathrm{~h}$, the number of surviving OHCs $(36.5 \pm 9.1)$ and IHCs $(11.2 \pm 3.4)$ in a section of $80 \mathrm{HCs}$ was significantly lower than the number of surviving OHCs $(59.0 \pm 2.1, p<0.0001)$ and IHCs $(18.1 \pm 2.7, p<0.0001)$ in untreated OCs (Fig. 3a). In OCs exposed to gentamicin and brimonidine, the number of surviving OHCs $(49.6 \pm 10.1)$ was significantly higher than the number of surviving $\mathrm{OHCs}$ that were exposed to gentamicin alone $(36.5 \pm 9.1, p<0.0001$; Fig. 3a). There were significantly fewer surviving OHCs in OCs exposed to gentamicin, brimonidine, and yohimbine (33.9 \pm 7.8$)$ versus those exposed to gentamicin and brimonidine $(49.6 \pm 10.1, p<0.0001$; Fig. 3a). Most of the OHCs survived in OCs that were exposed to gentamicin and brimonidine; the OCs showed few gaps in the normal ordered array of HCs (Fig. 3b). Notably, in OCs exposed to yohimbine, brimonidine, and gentamicin, multiple $\mathrm{OHCs}$ underwent apoptosis, creating a gap in the normal ordered array of HCs (Fig. 3b). Hence, the protective effect of brimonidine on OHCs was inhibited by yohimbine. 
The Expression Levels of the $\alpha_{2 a}$-AR Protein Are

Not Changed by Exposure for $8 \mathrm{~h}$ to Brimonidine,

Yohimbine, and/or Gentamicin

The effects of brimonidine are mediated by the $\alpha_{2}$-AR . Interestingly, brimonidine is a strong agonist of the $\alpha_{2 \mathrm{a}^{-}}$ AR subtype but only a weak agonist of the $\alpha_{2 b}$ and the $\alpha_{2 c^{-}}$ AR subtypes [Peltonen et al., 1998]. We investigated the expression levels of the $\alpha_{2 a}$-AR protein to examine whether brimonidine upregulated $\alpha_{2 \mathrm{a}}$-AR expression. Exposure of OCs to $400 \mu \mathrm{M}$ of gentamicin, $100 \mu \mathrm{M}$ of brimonidine, and/or $10 \mu \mathrm{M}$ of yohimbine for $8 \mathrm{~h}$ did not affect the expression level of the $\alpha_{2 \mathrm{a}}$-AR protein (Fig. $4 \mathrm{a}, \mathrm{b}$ ).

\section{Brimonidine Does Not Significantly Change the}

Activation Levels of the Erk1/2 and Akt Proteins after 8 or 24 h of Exposure

Phosphorylation of Erk1/2 mediates HC survival [Bas et al., 2012; Kurioka et al., 2015], and brimonidine pro- motes Erk1/2 phosphorylation [Fujita et al., 2013; Lai et al., 2002]. We did not find any significant changes in the phosphorylation levels of Erk1/2 after exposure to gentamicin. After $8 \mathrm{~h}$, the activation level of the Erk $1 / 2$ protein was lower in OCs exposed to gentamicin compared to the control group ( $p>0.99$; Fig. 5a, b). Brimonidine showed a tendency to increase the activation level of the Erk $1 / 2$ protein (Fig. 5a, b). After exposure to brimonidine for $24 \mathrm{~h}$, the Erk1/2 protein activation level showed the same tendency as after treatment for $8 \mathrm{~h}$ (Fig. 5c, d).

Our group recently showed that Akt is involved in HC survival [Brand et al., 2015], and brimonidine leads to the phosphorylation of Akt [Kim et al., 2007; Lai et al., 2002; Tatton et al., 2001]. However, in this study, the increases in the phosphorylation levels of Akt after exposure to brimonidine were not statistically significant. The activation level of the Akt protein was reduced after $8 \mathrm{~h}$ in OCs exposed to gentamicin alone compared to the control group

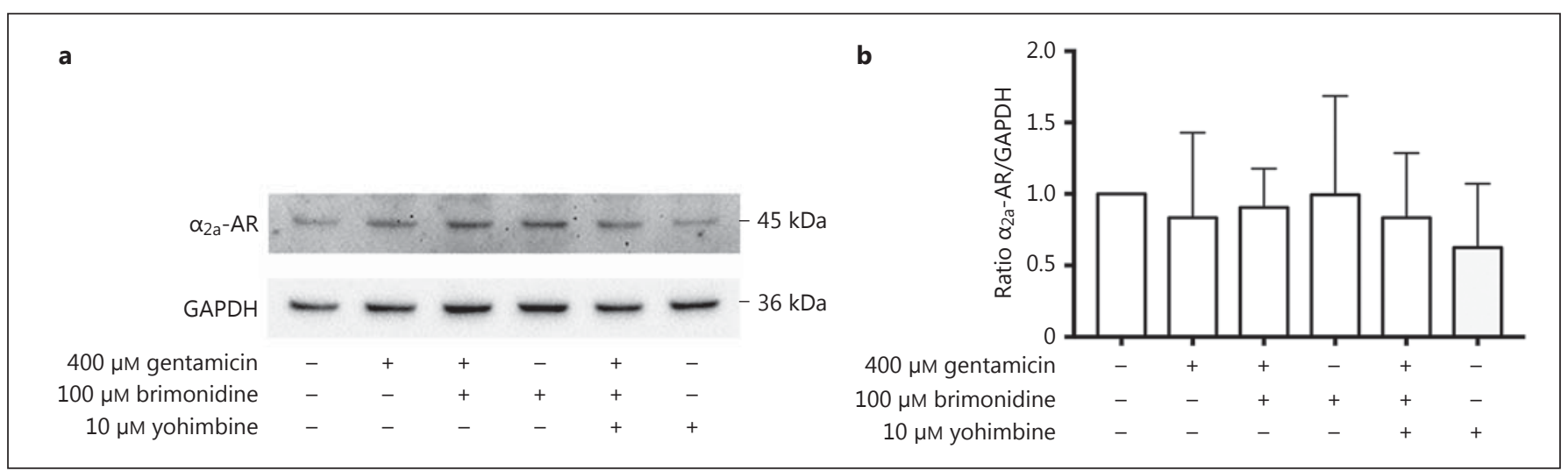

Fig. 4. Expression levels of the $\alpha_{2 a}$ adrenergic receptor $\left(\alpha_{2 a}-A R\right)$ protein are not changed by organ of Corti (OC) explant exposure to brimonidine, yohimbine, and/or gentamicin. a Representative Western blot showing the expression of $\alpha_{2 a}$-AR. b Quantification of the $\alpha_{2 \mathrm{a}}$-AR/GAPDH ratio. There was no significant difference in the $a_{2 \mathrm{a}}-\mathrm{AR} / \mathrm{GAPDH}$ ratio in explants exposed to the indicated concentrations of gentamicin, brimonidine, and/or yohimbine versus untreated explants (control). Two independent experiments were performed with $n=6-7$ pooled explants for each condition. The explants were exposed to gentamicin, brimonidine, and/or yohimbine for $8 \mathrm{~h}$. GAPDH was used as a loading control. Values are shown as means + SDs.
Fig. 5. The effects of brimonidine, yohimbine, and gentamicin on the levels of activated Erk and Akt proteins. a Representative Western blots of p-Erk and total Erk. $\mathbf{b}$ Quantification of the p-Erk/Erk ratio after exposure to the indicated concentrations of gentamicin, brimonidine, and/or yohimbine for $8 \mathrm{~h}$. Exposure to brimonidine did not significantly change the levels of activated Erk1/2. Two independent experiments were performed with $n=6-7$ pooled explants for each condition. c Representative Western blots of p-Erk and total Erk. d Quantification of the p-Erk/Erk ratio after exposure to the indicated concentrations of gentamicin, brimonidine, and/or yohimbine for $24 \mathrm{~h}$. Three independent experiments were performed with $n=6-7$ pooled explants for each condition. e Rep- resentative Western blots of $\mathrm{p}$-Akt and total Akt. $\mathbf{f}$ Quantification of the p-Akt/Akt ratio after exposure to the indicated concentrations of gentamicin, brimonidine, and/or yohimbine for $8 \mathrm{~h}$. Brimonidine did not significantly change the levels of activated Akt protein. Two independent experiments were performed with $n=$ 6-7 pooled explants for each condition. g Representative Western blots of p-Akt and total Akt. h Quantification of the p-Akt/Akt ratio after exposure to the indicated concentrations of gentamicin, brimonidine, and/or yohimbine for $24 \mathrm{~h}$. Three independent experiments were performed with $n=6-7$ pooled explants for each condition. For each membrane, GAPDH was used as a loading control. Values are shown as means + SDs.

(For figure see next page.) 
a

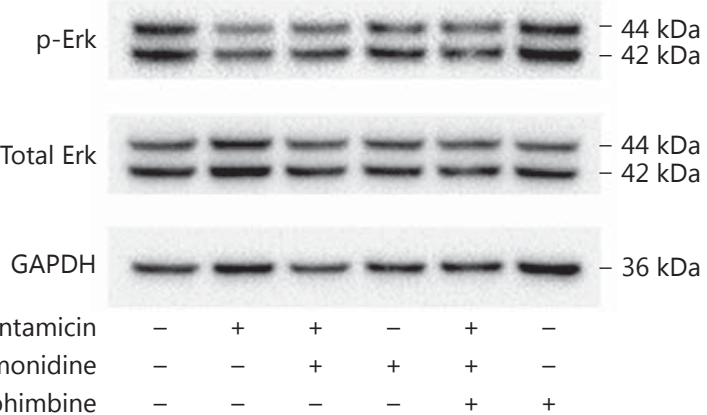

c

$\begin{array}{rllllll}400 \mu \mathrm{M} \text { gentamicin } & - & + & + & - & + & - \\ 100 \mu \mathrm{M} \text { brimonidine } & - & - & + & + & + & - \\ 10 \mu \mathrm{M} \text { yohimbine } & - & - & - & - & + & +\end{array}$

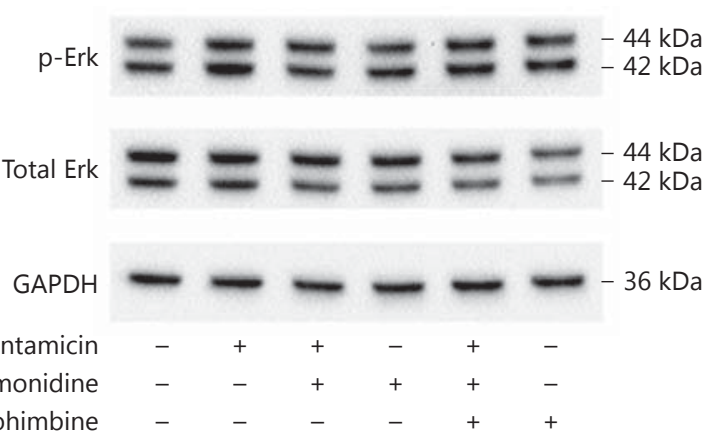

e

$400 \mu \mathrm{M}$ gentamicin $100 \mu \mathrm{M}$ brimonidine

$10 \mu \mathrm{M}$ yohimbine

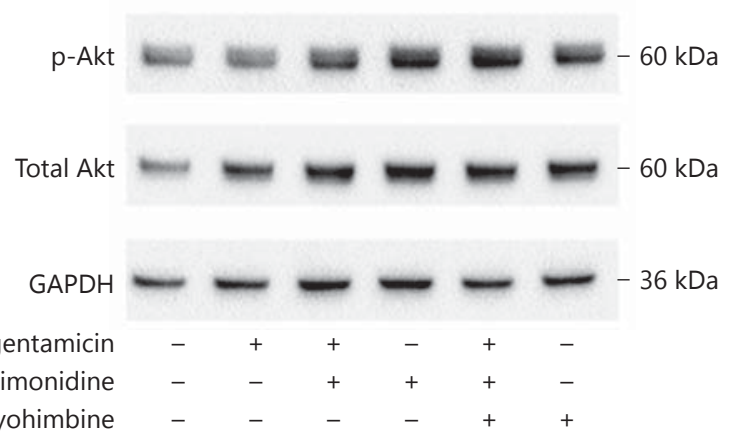

g

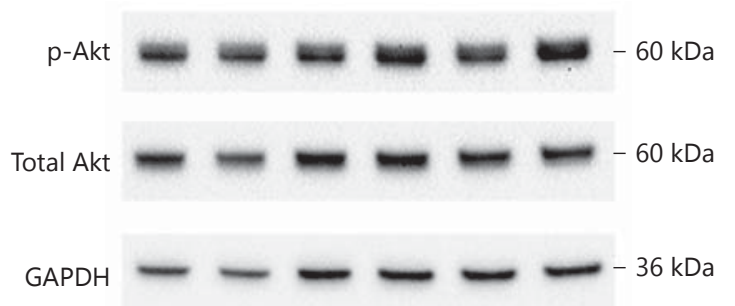

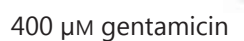
$100 \mu \mathrm{M}$ brimonidine

$10 \mu \mathrm{M}$ yohimbine b

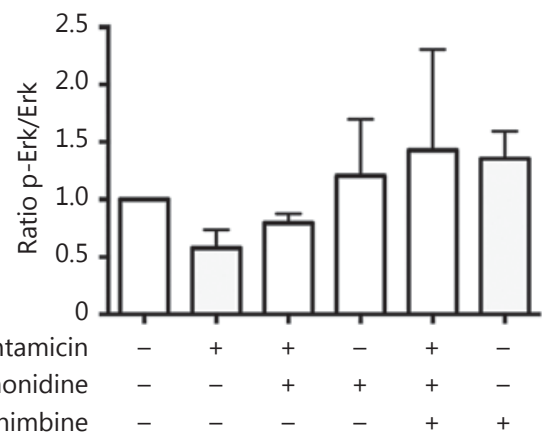

d

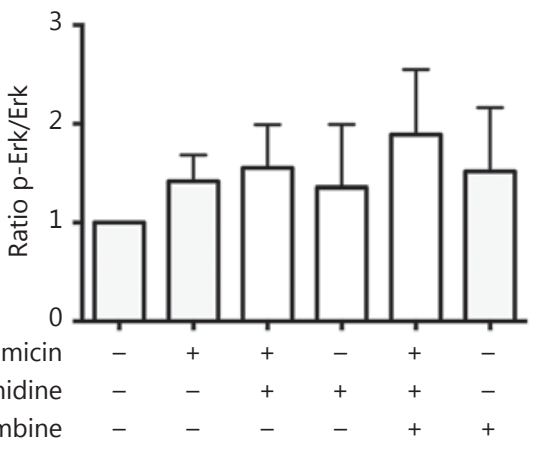

f

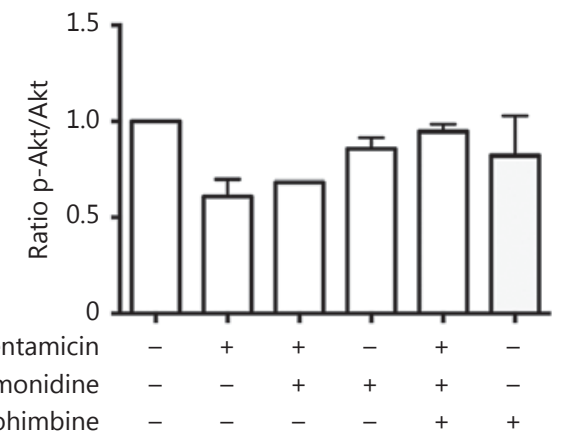

h

$400 \mu \mathrm{M}$ gentamicin $100 \mu \mathrm{M}$ brimonidine $10 \mu \mathrm{M}$ yohimbine

$400 \mu \mathrm{M}$ gentamicin $100 \mu \mathrm{M}$ brimonidine

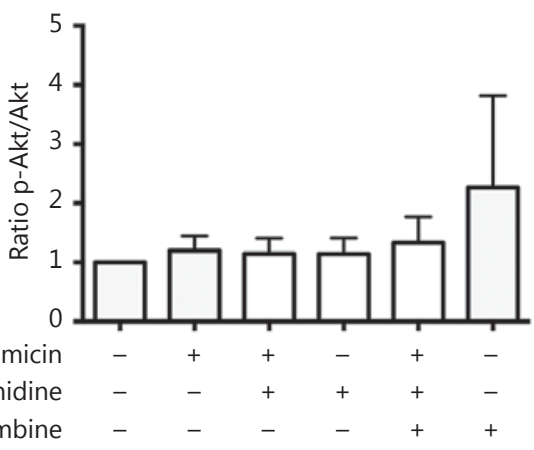

Brimonidine Protects against GentamicinInduced HC Loss in vitro
Audiol Neurotol 2017;22:125-134

DOI: $10.1159 / 000479218$
131 
( $p=0.08)$; however, this result did not reach statistical significance (Fig. 5e, f). Brimonidine showed a tendency to increase the Akt protein activation level (Fig. 5e, f). After $24 \mathrm{~h}$ of exposure to brimonidine, we observed no further increase in Akt protein activation (Fig. $5 \mathrm{~g}, \mathrm{~h}$ ).

\section{Discussion}

Previous studies have shown that IHCs and OHCs in the rat neonatal cochlea express all $3 \alpha_{2}$-AR subtypes [Cai et al., 2013b]. The $\alpha_{2}$-AR mediates the physiological response to the catecholamines adrenaline and noradrenaline, and adrenergic innervation of the OC has been shown [Drescher et al., 2006]. However, the role of the $\alpha_{2}$-AR in HCs is currently unknown. Because we observed that brimonidine has protective effects in the visual system and in the spiral ganglion, we wanted to investigate whether brimonidine had protective effects on auditory HCs in vitro.

\section{Brimonidine Protects OHCs from Gentamicin-}

Induced Toxicity

Exposure to gentamicin leads to a significant loss of IHCs and OHCs. The gentamicin-induced damage to HCs that we observed was similar to that observed in our earlier study [Glutz et al., 2015] and in studies by other groups [Battaglia et al., 2003; Chung et al., 2006]. In vitro aminoglycoside toxicity is a common model for investigating ototoxicity, and several recent studies using gentamicin revealed critical intracellular events that mediate HC survival [Battaglia et al., 2003; Brand et al., 2015; Caelers et al., 2010; Chung et al., 2006].

Interestingly, we found that brimonidine itself significantly reduced $\mathrm{OHC}$ loss in OC samples that were exposed to gentamicin plus brimonidine compared to samples exposed only to gentamicin. Brimonidine showed a protective effect on OHCs but not on IHCs. The receptor density of all of the $\alpha_{2}$-AR subtypes are equal on OHCs and IHCs on P3 and P8 [Cai et al., 2013b]. We investigated $\mathrm{P} 5$ rats, and we assumed that the IHCs and OHCs had the same $\alpha_{2}$-AR density. One possible reason for the protective effect of brimonidine that we observed for OHCs but not IHCs is that IHCs were less damaged by gentamicin than OHCs. OHCs are often more sensitive to damage than IHCs, partly because of intrinsically greater susceptibility [Sha et al., 2001]. Furthermore, the activation of $G$ protein-coupled receptors has been shown to regulate diverse signaling pathways [Rosenbaum et al., 2009], and the availability of specific pathway components may differ between the auditory $\mathrm{HC}$ types.
There are no previous reports of brimonidine-induced intracellular events in auditory HCs. However, our results are in line with other studies that found brimonidine-mediated protection in distinct experimental injury models in the visual system [Kim et al., 2007; Knels et al., 2008; Lai et al., 2002]. The retina and the inner ear share some common characteristics: both have a neuroepithelial origin, both contain sensitive sensory cells together with supporting cells, and both have a highly organized microarchitecture. Therefore, the molecular mechanisms involved in brimonidine-mediated protection in the retina may be similar to those involved in HCs. Moreover, brimonidine acts as a neuroprotective agent for the spiral ganglion [Cai et al., 2013a], supporting the idea that brimonidine has protective activity in the inner ear.

Yohimbine, an $\alpha_{2}$-AR antagonist, blocked the protective effect of brimonidine. This is important because it suggests that the protective effect of brimonidine requires activation of the $\alpha_{2}$-AR and hence is mediated by the $\alpha_{2^{-}}$ AR itself. Cai et al. [2013a] found similar results in that yohimbine blocked the protective effects of brimonidine against glutamate and hydrogen peroxide toxicity. In the visual system, the protective effects of brimonidine were also blocked by yohimbine [Lai et al., 2002]. These observations raised the question of how to explain the observed protective effects of brimonidine and the relationship of these effects to $a_{2}$-AR. We investigated this, and discuss some possible mechanisms below.

\section{Brimonidine Does Not Change the Expression Level of} the $\alpha_{2}$-AR Protein

Brimonidine is a strong agonist of the $\alpha_{2 a}-A R$ and a weaker agonist of the $\alpha_{2 b}$-AR and the $\alpha_{2 c}$-AR [Peltonen et al., 1998]. To investigate whether brimonidine upregulated $\alpha_{2 \mathrm{a}}-\mathrm{AR}$, we investigated $\alpha_{2 \mathrm{a}}$-AR protein expression levels. Exposure of OCs to gentamicin, brimonidine, and/ or yohimbine for $8 \mathrm{~h}$ did not result in significant differences in the expression level of the $\alpha_{2 \mathrm{a}}$-AR protein in the different conditions, indicating that brimonidine neither upregulates nor downregulates the $\alpha_{2 \mathrm{a}}$-AR. Interestingly, Cai et al. [2013a] reported that after $24 \mathrm{~h}$ of exposure to hydrogen peroxide and brimonidine, the $a_{2 a}$-AR protein level was unchanged compared to the hydrogen peroxide-only treated groups, whereas after treatment with glutamate and brimonidine, the $\alpha_{2 a}$-AR level was upregulated compared to the glutamate-only groups. However, $\alpha_{2 \mathrm{a}}$-AR was not upregulated in the glutamate- and brimonidine-treated groups compared to the untreated control groups in the present study. This indicates that glutamate

Cortada/Levano/Bodmer 
downregulated the $\alpha_{2 \mathrm{a}}-\mathrm{AR}$ and that brimonidine did not significantly change the expression level of the $\alpha_{2 \mathrm{a}}$-AR protein. Taken together, our results and the results of Cai et al. [2013a] indicate that the effect of brimonidine is not mediated by a change in the expression level of the $\alpha_{2 a}$-AR protein.

\section{Brimonidine Does Not Significantly Change the}

Activation Levels of the Erk1/2 and Akt Proteins

Our experiments indicated that brimonidine does not significantly change the activation levels of the Erk1/2 protein. Because there was no statistically significant effect, our data does not clarify the effect of brimonidine on Erk1/2 activation. Therefore, we can only speculate about a p-Erk1/2-mediated protective effect on HCs. Erk1/2 plays a role in several cell functions [Kurioka et al., 2015; Lahne and Gale, 2008]. In HCs, Erk proteins are involved in both cell survival [Bas et al., 2012; Battaglia et al., 2003; Kurioka et al., 2015] and cell death [Kalinec et al., 2005; Lahne and Gale, 2008], playing a complex role in the regulation of distinct cellular responses. The reports of the effects of brimonidine treatment on Erk1/2 activation have been contradictory; some studies report that brimonidine increases p-Erk1/2 levels [Fujita et al., 2013; Lai et al., 2002], while others report that it decreases p-Erk1/2 levels [Cai et al., 2013a; Harun-Or-Rashid et al., 2015]. In our study, Erk1/2 activation levels were measured in whole OC explants, so it is not possible to tell whether Erk1/2 activation occurred in supporting cells, as reported by Lahne and Gale [2008] or in HCs themselves.

Brimonidine does not significantly change the activation levels of the Akt protein after 8 or $24 \mathrm{~h}$ of exposure. In the visual system, brimonidine activates the PI3 kinase pathway [Knels et al., 2008; Yukita et al., 2016] and also leads to Akt phosphorylation [Kim et al., 2007; Lai et al., 2002; Tatton et al., 2001]. Interestingly, the PI3 kinase pathway has been shown to mediate HC survival of gentamicin-induced toxicity [Chung et al., 2006]. Moreover, our group showed that Akt 2 and Akt 3 are involved in $\mathrm{HC}$ survival in response to gentamicin-induced toxicity [Brand et al., 2015]. Because of these contradictory results, it seems possible that $\mathrm{p}$-Erk1/2 and p-Akt might not be the direct targets of brimonidine.

In addition to these molecular mechanisms, the neuroprotective effects of brimonidine are also due to a reduction in NMDA-elicited intracellular signals [Dong et al., 2008; Han and Wu, 2002]. Notably, NMDA receptors in the cochlea may be involved in the ototoxic effects of aminoglycosides, and NMDA receptor antagonists attenuate these ototoxic effects [Basile et al., 1996; Nagy et al.,
2004]. Furthermore, brimonidine blocks increases in Bax, caspase-9, and caspase-3 [Cai et al., 2013a], abolishes the generation of reactive oxygen species [Knels et al., 2008], and upregulates antiapoptotic genes such as Bcl-2 [Lai et al., 2002]. Caspase inhibition, inhibition of reactive oxygen species, and a protective role of $\mathrm{Bcl}-2$ all promote $\mathrm{HC}$ survival [Cheng et al., 2005]. Brimonidine also upregulates growth factors [Gao et al., 2002; Kim et al., 2007; Lonngren et al., 2006], and growth factors attenuate noise-induced hearing loss and increase $\mathrm{OHC}$ survival [Shoji et al., 2000].

In our study, it is not clear which mechanisms are involved in the protective effects of brimonidine against gentamicin-induced toxicity in HCs. It is possible that these mechanisms act in concert to produce the observed effects or that there are as-yet undiscovered mechanisms or secondary messengers responsible for the otoprotective effects. Furthermore, the functional relevance and contribution of these protective mechanisms to $\mathrm{HC}$ survival may depend on the type of cellular injury and on the metabolic state of the cells. Future studies that investigate the precise molecular mechanisms underlying brimonidine's protective effect on HCs will increase our understanding of cell survival mechanisms in auditory HCs.

\section{Conclusions}

To the best of our knowledge, this is the first paper to study the effects of brimonidine on auditory HCs. We found that brimonidine can protect auditory HCs from gentamicin-induced ototoxicity in vitro and most likely does so through $\alpha_{2}$-AR signaling.

Very little is known about the role of the $\alpha_{2}$-AR in auditory HCs. Future research that investigates the precise molecular mechanisms that underlie the protective effect of $\alpha_{2}$-AR signaling in HCs may shed more light on our understanding of $\mathrm{HC}$ cell survival mechanisms. Finally, the $\alpha_{2}$-AR agonist brimonidine has a protective effect on HCs as well as on the spiral ganglion [Cai et al., 2013a], and it shows potential for the prevention and/or treatment of sensorineural hearing loss in the future.

\section{Disclosure Statement}

The authors declare no competing financial interests. 


\section{References}

- Bas E, van de Water TR, Gupta C, Dinh J, Vu L, Martinez-Soriano F, Lainez JM, Marco J: Efficacy of three drugs for protecting against gentamicin-induced hair cell and hearing losses. Br J Pharmacol 2012;166:1888-1904.

- Basile AS, Huang JM, Xie C, Webster D, Berlin C, Skolnick P: N-methyl-D-aspartate antagonists limit aminoglycoside antibiotic-induced hearing loss. Nat Med 1996;2:1338-1343.

- Battaglia A, Pak K, Brors D, Bodmer D, Frangos JA, Ryan AF: Involvement of ras activation in toxic hair cell damage of the mammalian cochlea. Neuroscience 2003;122:1025-1035.

Bielefeld EC, Henderson D: Influence of sympathetic fibers on noise-induced hearing loss in the chinchilla. Hear Res 2007;223:11-19.

-Brand Y, Levano S, Radojevic V, Naldi AM, Setz C, Ryan AF, Pak K, Hemmings BA, Bodmer $\mathrm{D}$ : All Akt isoforms (Akt1, Akt2, Akt3) are involved in normal hearing, but only Akt 2 and Akt 3 are involved in auditory hair cell survival in the mammalian inner ear. PLoS One 2015;10:e0121599.

-Caelers A, Radojevic V, Traenkle J, Brand Y, Bodmer D: Stress and survival pathways in the mammalian cochlea. Audiol Neurootol 2010; 15:282-290.

Cai J, Li J, Liu W, Han Y, Wang H: Alpha2-adrenergic receptors in spiral ganglion neurons may mediate protective effects of brimonidine and yohimbine against glutamate and hydrogen peroxide toxicity. Neuroscience 2013a;228:23-35.

Cai J, Li J, Mao Y, Bai X, Xu L, Wang H: Immunohistochemical localization of $\alpha_{2}$-adrenergic receptors in the neonatal rat cochlea and the vestibular labyrinth. J Mol Neurosci 2013b;51: 1010-1020.

Cheng AG, Cunningham LL, Rubel EW: Mechanisms of hair cell death and protection. Curr Opin Otolaryngol Head Neck Surg 2005; 13 : 343-348.

-Chung WH, Pak K, Lin B, Webster N, Ryan AF: A PI3K pathway mediates hair cell survival and opposes gentamicin toxicity in neonatal rat organ of Corti. J Assoc Res Otolaryngol 2006;7:373-382.

Daunt DA, Hurt C, Hein L, Kallio J, Feng F, Kobilka BK: Subtype-specific intracellular trafficking of $\alpha_{2}$-adrenergic receptors. Mol Pharmacol 1997;51:711-720.

-Dong CJ, Guo Y, Agey P, Wheeler L, Hare WA: a2 adrenergic modulation of NMDA receptor function as a major mechanism of RGC protection in experimental glaucoma and retinal excitotoxicity. Invest Ophthalmol Vis Sci 2008;49:4515-4522.

- Drescher MJ, Drescher DG, Khan KM, Hatfield JS, Ramakrishnan NA, Abu-Hamdan MD, Lemonnier LA: Pituitary adenylyl cyclase-activating polypeptide (PACAP) and its recep- tor (PAC1-R) are positioned to modulate afferent signaling in the cochlea. Neuroscience 2006;142:139-164.

Fujita Y, Sato A, Yamashita T: Brimonidine promotes axon growth after optic nerve injury through Erk phosphorylation. Cell Death Dis 2013;4:e763.

Gao H, Qiao X, Cantor LB, WuDunn D: Up-regulation of brain-derived neurotrophic factor expression by brimonidine in rat retinal ganglion cells. Arch Ophthalmol 2002;120:797803.

Glutz A, Leitmeyer K, Setz C, Brand Y, Bodmer D: Metformin protects auditory hair cells from gentamicin-induced toxicity in vitro. Audiol Neurootol 2015;20:360-369.

Grueb M, Bartz-Schmidt KU, Rohrbach JM: Adrenergic regulation of $\mathrm{CAMP} /$ protein kinase A pathway in corneal epithelium and endothelium. Ophthalmic Res 2008;40:322-328.

Han Y, Wu SM: NMDA-evoked $\left[\mathrm{Ca}^{2+}\right]$ i increase in salamander retinal ganglion cells: modulation by PKA and adrenergic receptors. Vis Neurosci 2002;19:249-256.

-Harun-Or-Rashid M, Diaz-DelCastillo M, Galindo-Romero C, Hallbook F: Alpha2-adrenergic-agonist brimonidine stimulates negative feedback and attenuates injury-induced phospho-ERK and dedifferentiation of chicken Müller cells. Invest Ophthalmol Vis Sci 2015;56:5933-5945.

-Harun-Or-Rashid M, Lindqvist N, Hallbook F: Transactivation of EGF receptors in chicken Müller cells by a2A-adrenergic receptors stimulated by brimonidine. Invest Ophthalmol Vis Sci 2014;55:3385-3394.

- Hawes BE, van Biesen T, Koch WJ, Luttrell LM, Lefkowitz RJ: Distinct pathways of Gi- and Gq-mediated mitogen-activated protein kinase activation. J Biol Chem 1995;270:1714817153.

Kalinec GM, Fernandez-Zapico ME, Urrutia R, Esteban-Cruciani N, Chen S, Kalinec F: Pivotal role of Harakiri in the induction and prevention of gentamicin-induced hearing loss. Proc Natl Acad Sci USA 2005;102:1601916024.

Kim HS, Chang YI, Kim JH, Park CK: Alteration of retinal intrinsic survival signal and effect of a2-adrenergic receptor agonist in the retina of the chronic ocular hypertension rat. Vis Neurosci 2007;24:127-139.

Knels L, Worm M, Wendel M, Roehlecke C, Kniep E, Funk RH: Effects of advanced glycation end products-inductor glyoxal and hydrogen peroxide as oxidative stress factors on rat retinal organ cultures and neuroprotection by UK-14,304. J Neurochem 2008; 106 : 1876-1887.

-Kurioka T, Matsunobu T, Satoh Y, Niwa K, Endo S, Fujioka M, Shiotani A: ERK2 mediates in- ner hair cell survival and decreases susceptibility to noise-induced hearing loss. Sci Rep 2015;5:16839.

Lahne M, Gale JE: Damage-induced activation of ERK1/2 in cochlear supporting cells is a hair cell death-promoting signal that depends on extracellular ATP and calcium. J Neurosci 2008;28:4918-4928.

Lai RK, Chun T, Hasson D, Lee S, Mehrbod F, Wheeler L: Alpha-2 adrenoceptor agonist protects retinal function after acute retinal ischemic injury in the rat. Vis Neurosci 2002; 19:175-185

Lonngren U, Napankangas U, Lafuente M, Mayor S, Lindqvist N, Vidal-Sanz M, Hallbook F: The growth factor response in ischemic rat retina and superior colliculus after brimonidine pre-treatment. Brain Res Bull 2006;71: 208-218.

-MacDonald E, Kobilka BK, Scheinin M: Gene targeting - homing in on $a_{2}$-adrenoceptorsubtype function. Trends Pharmacol Sci 1997; 18:211-219.

-Nagy I, Bodmer M, Brors D, Bodmer D: Early gene expression in the organ of Corti exposed to gentamicin. Hear Res 2004;195:1-8.

-Navaratnam DS, Su HS, Scott SP, Oberholtzer JC: Proliferation in the auditory receptor epithelium mediated by a cyclic AMP-dependent signaling pathway. Nat Med 1996;2:1136-1139.

Peltonen JM, Pihlavisto M, Scheinin M: Subtypespecific stimulation of $\left[{ }^{35} \mathrm{~S}\right] \mathrm{GTP} \gamma \mathrm{S}$ binding by recombinant $\alpha_{2}$-adrenoceptors. Eur J Pharmacol 1998;355:275-279.

Rosenbaum DM, Rasmussen SG, Kobilka BK: The structure and function of G-protein-coupled receptors. Nature 2009;459:356-363.

Sha SH, Taylor R, Forge A, Schacht J: Differential vulnerability of basal and apical hair cells is based on intrinsic susceptibility to free radicals. Hear Res 2001;155:1-8.

Shoji F, Miller AL, Mitchell A, Yamasoba T, Altschuler RA, Miller JM: Differential protective effects of neurotrophins in the attenuation of noise-induced hair cell loss. Hear Res 2000;146:134-142.

- Tatton WG, Chalmers-Redman RM, Tatton NA: Apoptosis and anti-apoptosis signalling in glaucomatous retinopathy. Eur J Ophthalmol 2001;11(suppl 2):S12-S22.

World Health Organization: WHO global estimates on prevalence of hearing loss. 2012. http://www.who.int/pbd/deafness/estimates/ en/.

Yukita M, Omodaka K, Machida S, Yasuda M, Sato K, Maruyama K, Nishiguchi KM, Nakazawa T: Brimonidine enhances the electrophysiological response of retinal ganglion cells through the Trk-MAPK/ERK and PI3K pathways in axotomized eyes. Curr Eye Res 2017;42:125-133. 